\title{
Towards a High-level Service Delivery Architecture for One-Stop Government (OSG)
}

Thi Thu Huong Nguyen

School of Information Systems

Queensland University of Technology

Brisbane, Australia

Email: t340.nguyen@connect.qut.edu.au

Mustafa Hashmi

'Data61, CSIRO, Dutton Park, Brisbane Queensland

${ }^{2}$ Federation University (FedUni), Brisbane, Queensland

Email: mustafa.hashmi@data61.csiro.au

Mary Tate

School of Information Systems

Victoria University of Wellington

Wellington, New Zealand

Email: mary.tate@vuw.ac.nz

\section{Abstract:}

The work on the service delivery in the context of one-shop government architecure is far from being mature. Although the understanding on various levels of complexity of e-government service delivery has been addressed, many extant versions of the service delivery architectures themselves are dated and do not reflect the potential contribution of new technologies. In this work-in-progress paper, we report ongoing work and propose an updated service delivery architecutre comprising five distinct layers. The proposed architecture addresses some important challenges, and suggests areas for future research as outlined in the paper.

Keywords: One-stop government, High-level architecture, Maturity models, Architecture layers 


\section{INTRODUCTION}

The potential of one-stop government has captured the interest of officials and citizens for several decades and has been considered as a key factor for public administration's development (Kubicek \& Hagen, 2000). One-stop government (hereafter, OSG) is a digital presence of government mainly in a form of well-designed website supported by other services, and provides integrated access to multiple public services for different groups of customers all in one interface. OSG applies initiatives from information and communication technologies (Dias \& Rafael, 2007) in order to transform government administration processes and allow more active participation from citizens (Lee, 2010). Many public services are integrated into OSG, including paying taxes online, gathering improvement grants information or accessing to a number of health services, and many other services (Pollitt, 2003).

A key feature of OSG is the integration of information and transactions across agencies, so citizens and businesses can minimise interaction with government while having their services delivered in a better and faster way (Ebrahim \& Irani, 2005). From a government's perspective, there is also cost saving in terms of human resources and administration management because services are designed in an automated manner, and require fewer staff and processes. In Australia, OSG solutions are provided at different levels of government and are showing great benefits for all types of users. For example, MyGov ${ }^{1}$ is an OSG at national level, in which everyone can use the portal, either citizens or immigrants. At state level, New South Wale's OneGov² (NSW's OneGov) can be considered as a good example with a wide range of public services offered.

However, along with opportunities to transform the way government implements traditional public services, OSG also poses many issues in terms of architecture as OSG is still evolving and has not yet reached a mature state (Lofstedt, 2012). According to Ebrahim and Irani, there is a lack of architecture frameworks for OSG as many governments are still in the early stages of implementing integrated digital portals of public services (Ebrahim \& Irani, 2005). In addition, OSG systems can be fragmented, as many services are not fully integrated. Low productivity, inconsistent workflow and underperforming systems are consequences of these existing problems (Keld, 2017). Therefore, a more robust architecture is necessary to better design an OSG portal (Ebrahim \& Irani, 2005).

This work-in-progress study of OSG's high-level architecture aims to provide governments with overall guidelines to improve public services' core design by applying new technologies, for example cloud computing and data mining, $\mathrm{AI}$, and analytics. In the rest of this paper, we first review previous studies relevant to the topic, including insights about the evolution and maturity of OSG. We then develop a high-level architecture model for OSG, with areas for future research. This is followed by a discussion and conclusion.

\section{LITERATURE REVIEW}

\subsection{OSG maturity models in relation with $O S G$ architecture}

The adoption of OSGs can improve the time taken to deliver of public services to citizens and businesses, while simplifying bureaucratic procedures, reducing transaction costs and enhancing transparency for governments (Saha, 2012). However, in order to achieve this quality of services, OSGs have been undergoing numerous changes in terms of evolution stages and high-level architectures. The evolution of the service capability of OSGs has close relationship with the underlying architectural structure. In each stage of the evolution, OSGs have reached new capabilities by adopting new technology initiatives. In 2000, Baum and Di Maio introduced first framework of OSG's evolution stages including web presence, interaction, transaction and transformation (Baum \& Di Maio, 2000; Layne \& Lee, 2001). Later, Layne and Lee proposed a four-stage model: catalogue, transaction, vertical integration, and horizontal integration (Layne \& Lee, 2001). Their model is the most widely cited when discussing the development phases of OSG (Debri \& Bannister, 2015). Siau and Long (2005), on the other hand, extended the work of Baum and Di Maio by adding e-democracy as the maturity stage of OSG to open up the possibility for political participation. The integration of e-democracy seems to be the highest maturity level of OSG until a new trend which allow consumers (citizens and businesses) to have more power in resolving public problems was proposed by (Linders, 2012). This trend raised a new concept in which OSG is no longer in its form of e-government but rather evolves to a we-government. With the notion of a platform that treats citizens and businesses not as consumers but partners, Linders

\footnotetext{
1 https:/ / my.gov.au/ LoginServices/ main/ login?execution=e1s1 (retrieved 21st May 2018).
}

2 https:// onegov.nsw.gov.au/ new/ (retrieved 21st May 2018). 
emphasized the importance of citizen coproduction with government's efforts in tackling public issues. Thus, this concept can open new approach for OSG's architecture design not only from the interface layer point of view but also for other core layers.

\subsection{Challenges of OSG}

Implementations of OSG still appear to be far from reaching their potential because there are many that challenges need to be addressed. Reasons are varied, and in this section, we discuss three of the most challenging problems for the development of OSG.

Technology capabilities are changing rapidly. OSG is not limited to a particular technology or framework from the past; rather there are rich opportunities to utilize contemporary technologies into OSG's architecture. Therefore, architectures need to be regularly reviewed, For example, many studies from the 2000's (Baum \& Di Maio, 2000; Siau \& Long, 2005) on OSG's stage model concentrate on web services as the platform for government's portal and have not taken into account the application of other dominant technologies, for example: cloud computing, AI, data analytics and so on (Debri \& Bannister, 2015). These technologies have been readily embraced by the private sector to improve service delivery. Tech giants such as Facebook and Amazon use predictive analytics, AI agents, and other tools. People expect public services to keep pace. In some respects, this is the case. Cloud computing, for example has changed the way data is stored by leveraging its capability to save a great amount of information with less investment on capital for infrastructure, installation as well as human resources (J adeja \& Modi, 2012). This technology is widely used by public sectors around the world (Wyld, 2010), and has become an essential component of OSG solutions. Additionally, the use of big-data in recent years is becoming extremely important in government contexts. Big data offers the opportunity for significant contributions to improving transparency, citizen's well-being, economic growth and national security (Kim, Trimi, \& Chung, 2014). With benefits from big-data, the Australian government has run a program called Government 2.0 in which offering public access to government data and enabling web-based automation tools (http:// data.gov.au) with the aim of better utilising government data resources (Kim et al., 2014). Simultaneously, data mining, another application of big-data, with its predictive analytics technique can help to translate a massive amount of data into recommender systems (Kim et al., 2014). This can be used (for example) to predict people at risk of becoming homeless, or needing other forms of public assistance. However, both privacy and security issues, and the scope of integration required across multiple agencies are challenges in the public sector context.

Data analytics raise major privacy concerns. Secondly, although predictive data mining can greatly benefit the decision making process of either private and public sector, the activities of extracting knowledge from massive databases can be a target for cyber-attacks (Malik, Ghazi, \& Ali, 2012). Accidently sharing the personal details of hundreds of customers of Centrelink in Australia is a bitter example of a security breach ${ }^{3}$. Internationally, other breaches have been reported ${ }^{4}$. As citizen data is increasingly shared and integrated, and stored in the cloud, the security of OSGs has become a major concern. Data security in cloud technology has become a pain point due to data loss, phishing and other digital criminal activities (Dillon, Wu, \& Chang, 2010). Essentially, the technical problems of security models (Lam, 2005), high cost of security applications and solutions (Lambrinoudakis, Gritzalis, Dridi, \& Pernul, 2003), high costs of security infrastructure, and lack of risk management security program (Ebrahim \& Irani, 2005) are other challenges in building more trusted system for OSG. As many public enterprises are now adopting new technologies, improved security measures and protection of citizen data is a fundamental requirement for OSGs.

Integration across agencies and levels of government is difficult. Thirdly, the aim of OSG's maturity models is to achieve a fully integrated system with cohesive and seamless public services for that is citizen (not agency) centric (Lam, 2005). However, many OSG solutions fall well short of this vision. One reason is that the structure of government agencies makes OSG integration hard to implement (2005). OSG at national level is different from state level and local level in a number of services integrated, form of services, security system, data collection methods, and so on. Lam (2005) has collected and analysed previous studies, then indicated that strategy, technology, policy and organisations are four main barriers for public services integration. Amongst various barriers, the author has shown that the lack of architecture interoperability is considered as "a major reason for failure in application integration", and it comes from the dissimilarity technology platforms, the closed design of applications, the disparity of programming framework. Lam also pointed out that beside

\footnotetext{
${ }^{3}$ https:// www.smh.com.au/ public-service/ centrelink-apologises-for-new-privacy-breach-20161101gsflcp.html (retrieved 13J une 2018).

${ }^{4}$ https:// www.bbc.com/news/ world-us-canada-33017310 (retrieved 12 J une 2018).
} 
architectural related issues, incompatible technical standards can also act as obstacles to integration. Equally important, even when governments prioritize compatible technical standards, other issues will occur, for example: differences in methodologies and modelling standards. Therefore, a standardised enterprise system and common technologies are desired to improve the use of OSG in public sector.

\subsection{Previous Enterprise Architecture Frameworks for e-Government}

Enterprise Architecture (EA) frameworks are used to manage the ongoing process of building the ability to tackle complexity, with the key goal of developing and sustaining coherent enterprises (Saha, 2012). Frequently, five layers are used, including: business architecture, process architecture, integration architecture, software architecture and technology (or infrastructure) layer (Winter \& Fischer, 2006). We note that vertically, OSGs s at different level, for example: national level, state level, and local level have different services packages, technologies, data collection methods, and so on. Horizontally, different departments also have different approaches and points of view about OSG. However, despite of these discrepancies, there should be a common architectural framework to guide the development of layers, processes and target results for each layer (Ebrahim \& Irani, 2005). Surprisingly, throughout the development of OSG, there are a few studies that develop architectural frameworks for OSG.

We draw from previous studies of enterprise architecture for e-government. Saha (2007) introduced four architectural domains specifically for the government context including business architecture, data/information architecture, application architecture and technical architecture. This was later expanded by adding policy and strategy architecture domains (Saha, 2012). Their work offer detailed practices for each layer-and provide the inter-relationship between layers, and build conditions for these layers to support each other. When government agencies are able to identify their business models for their public services as a whole, they can also make decision on technologies that best suit the development for those services. One of the most cited paper on e-government architecture is the work of Ebrahim and Irani (2005). The architecture introduced in their work consists of four layers named: access layer, e-government layer, e-business layer, infrastructure layer. Although this study is more than ten years old and has been extensively cited, subsequent studies have focussed primarily on the challenges and barriers to implementation (Lam, 2005) rather than on extending the framework. We were not able to identify any follow-up studies that elaborated aspects of the proposed e-government architecture itself. Understanding of the various levels of complexity of e-government services has progressed, but the service delivery architecture itself has not been updated. It is therefore timely to update and elaborate the architectures required for delivering e-government services at various levels of complexity. We use this work as a foundation for our architecture.

Our core study will apply the outcomes from previous researchers on enterprise architecture (EA) and its component layers to improve design thinking for OSG's high-level architecture-so it will be more appropriate for the current state of OSG services, systems and technology.

\section{A HIGH LEVEL ARCHITECTURAL FRAMEWORK}

The architectural framework that we introduce in this paper, presented in Figure 1, includes five distinct layers: interface layer, one-stop government layer, integration and personalization layer, business layer, and infrastructure layer. Each layer has an inter-relationship with the others, demonstrated by two-way arrows.

The interface layer is similar to the access layer in Ebrahim and Irani's (2005) framework, and allows OSG's users to interact with the portal. In this layer, users are classified into customers (individuals and businesses) and providers (governments and non-public providers). The sub-layer that contains channels divided into traditional and digital streams. While the traditional stream presents direct and manual methods, the digital stream presents possible online platforms, for example: web presence, mobile application. However, there is no limitation in the forms of digital channels as they can expand to social media, market place, smart TV, and other technology platforms that are applicable for OSG integration.

The One-stop-government layer contains two sub-layers. The first is a customer personal page and the second is a service layer. Within this layer, after accessing from interface layer into their personal pages, customers are able to choose their target services. There are two streams of services. The first stream includes services that are directly integrated and fulfilled by the portal, without the need to exchange data or pass control to other agencies. For example, vehicle licence renewal service is available directly 
on the NSW OSG5. On the other hand, the second stream requires redirection to other digital pages, for example: Australian OSG at national level, myGov can lead users to websites of ATO, Centrelink, Medicare, and other services websites ${ }^{6}$. This can be done with varying levels of sophistication. Sometimes, the integration and communication is fully or partly automated, at other times, the user themselves needs to initiate the integration of services from several agencies.

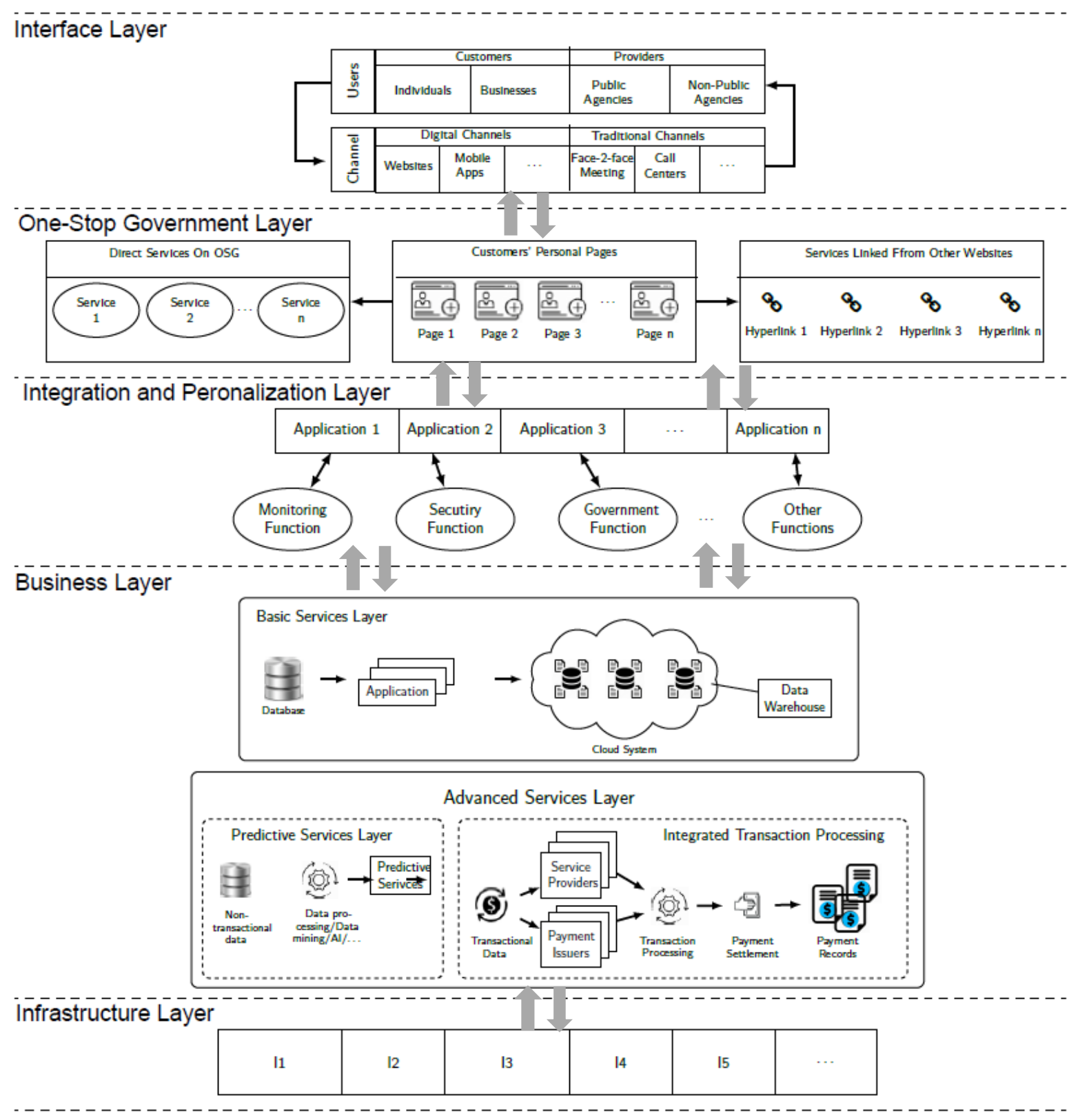

Figure 1: An architectural framework for One-stop Government (OSG)

The Integration and personalization layer plays a role of an intermediary, which connects front-end layers (interface and one-stop government layer) to back-end layers. This also serves as a protection wall for data and information in business layer. This layer can deliver agility, cost efficiency and integration (Winter \& Fischer, 2006). This layer developed in response to the problem of integrating services from multiple agencies. Furthermore, as this layer sits between the front-end and back-end layers it can significantly improve the protection of the data and digital infrastructure layer, because to reach these layers an external entity would need to interact thorough this intermediary layer. This layer can also serve as a placeholder for extended security applications and solutions. A major contribution of this layer is the ability to manage the data integration required to respond to a customer service need on an

${ }^{5}$ https:// www.onegov.nsw.gov.au/gls_portal/snsw/ Renew (retrieved 21st J une 2018).

${ }^{6} \mathrm{https}: / /$ my.gov.au/LoginServices/ main/login? execution=e1s1 (retrieved 21st J une 2018). 
secure, individual, just-in-time, need-to-know basis, reducing the risk of security breaches. It can also manage the process of seeking customer permission for data sharing, if required. In terms of mechanisms, it is created by combining multiple application each performing a particular function. For example, one application can have monitoring function while the other is implementing security function, or governance function. The set of applications and functions are not limited, they depend on target of each government agency and their technology capabilities.

The business layer is similar to Ebrahim and Irani's (2005) framework but different in components and structures. The special characteristic of this layer lies in its two sub-layers: (a) basic services and (b) advanced services. The basic service layer is responsible for storing data and carrying out services for users. Individual services can be siloed, and can be the responsibility of single agencies, the OSG functionality is achieved based on how these services can be integrated by the personalization and integration layer. The adoption of cloud computing technology will help governments to store a massive amount of information from simple database system. An example is that users of myGov are able to create accounts with personal information, which will be saved in this basic service layer with cloud computing technology. Added value is provided by the advanced services layer, which utilizes emerging technologies to deliver more sophisticated services, for example: generating predictive services for users, or processing the payment transaction. The benefits of predictive services can be enormous. It can help governments to identify services that are most accessed by users, then analyse the quality of the services to improve and innovate if necessary. For example, predictive service could be used when a user is receiving income support for job seekers, they can receive targeted advertisements related to job opportunities. Chatbots can potentially be a new advantage from using data mining technique. The payment/ transaction services layer is where transactional requests are handled. The mechanism behind this needs the involvement of both service providers (government agencies and non-public agencies) and payment issuers (e.g. banks). Basically, after receiving transactional data, these two entities will proceed the transaction to settle the payments, then record payment results as a final step. The advanced services layer manages secure transactions with other entities, and manages the creation of benefits from basic services, by using artificial intelligence, analytics, and other techniques.

Lastly, the infrastructure layer provides foundational technologies for all previous layers, for example: application server, hardware and operation system (Ebrahim \& Irani, 2005). Standard network and communication infrastructure should be integrated properly and take into account the revolution in technologies from other layers, so they can work smoothly and at the same time be prepared for changes in the future (Ebrahim \& Irani, 2005).

\section{CONCLUSIONS AND FUTURE OPPORTUNITIES}

As society is modernizing, citizens and businesses are expecting governments to have more innovative ideas to improve the transparency and convenience of public services. At the same time, governments also want to strengthen relationships with citizens and businesses, as well as reduce the time, money and resources required to deliver public services. With the growth of technology, OSG has emerged as the win-win solutions for the expectations from both customers and governments.

As OSG is now still far from reaching its maturity level, three main challenges have been identified, including the limitation in using contemporary technologies; the risk of data intrusion; and the difficulty of OSG integration. Our framework addresses the major challenges of OSG architecture. Our contributions are as follows. Based on the work of Ebrahim and Irani (2005), we designed a new architecture with five layers, including interface layer, one-stop government layer, integration and personalization layer, business layer and infrastructure layer. Integration and personalization is a new layer that plays a role of an intermediary wall between front-end system (the first two layers) and the back-end system (the last two layers) to protect, monitor and control data and all business activities. This provides a layer that can manage privacy and security concerns, traverse multiple agencies, request services, and manage communication with customers. It also allows OSG solutions to be developed incrementally for specific customer segments without solving the entire problem of service integration at once. Our enhanced business layer combines the use of cloud computing, predictive data mining/ AI, and payment transaction processing. It is extensible, and provides benefit for basic service delivery. Although we provide a way forward for the three afore-mentioned challenges, this is still a work-inprogress, with opportunities for other improvements. Our architecture is fairly high-level, and can be extended to provide more detail. With the rapid change of technology, OSG maturity models should be continuously updated as the purposes and functions of OSG are also changing. In addition, a major area for further research in the development of high-level process models for OSG. Our high-level 
architecture can open opportunities for mapping out detailed processes in the personalization and business layers in particular. Finally, a set of new challenges should be detected so the next architectural framework can come up with ideas to have more improvements and innovations.

\section{References}

Baum, C., \& Di Maio, A. (2000). Gartner's four phases of e-government model. Gartner Group, 12.

Debri, F., \& Bannister, F. (2015). E-government stage models: A contextual critique. Paper presented at the System Sciences (HICSS), 2015 48th Hawaii International Conference on.

Dias, G. P., \& Rafael, J . A. (2007). A simple model and a distributed architecture for realizing one-stop e-government. Electronic Commerce Research and Applications, 6(1), 81-90.

Dillon, T., Wu, C., \& Chang, E. (2010). Cloud computing: issues and challenges. Paper presented at the Advanced Information Networking and Applications (AINA), 2010 24th IEEE International Conference on.

Ebrahim, Z., \& Irani, Z. (2005). E-government adoption: architecture and barriers. Business process management journal, 11(5), 589-611.

J adeja, Y., \&Modi, K. (2012). Cloud computing-concepts, architecture and challenges. Paper presented at the Computing, Electronics and Electrical Technologies (ICCEET), 2012 International Conference on.

Keld, P. (2017). Realizing e-government benefits with minimal capabilities. Transforming Government: People, Process and Policy, 11(2), 262-285.

Kim, G.-H., Trimi, S., \& Chung, J.-H. (2014). Big-data applications in the government sector. Communications of the ACM, 57(3), 78-85.

Kubicek, H., \&Hagen, M. (2000). One stop government in Europe: an overview, in Hagen, M., Kubicek, H.(Eds). One Stop Government in Europe. Results from 11 National Surveys. Bremen, University of Bremen. 1-36.

Lam, W. (2005). Barriers to e-government integration. Journal of Enterprise Information Management, 18(5), 511-530.

Lambrinoudakis, C., Gritzalis, S., Dridi, F., \& Pernul, G. (2003). Security requirements for e-government services: a methodological approach for developing a common PKI-based security policy. Computer Communications, 26(16), 1873-1883.

Layne, K., \& Lee, J . (2001). Developing fully functional E-government: a four stage model. Government Information Quarterly, 18(2), 122-136. doi:10.1016/S0740-624X(01)00066-1

Lee, J . (2010). 10year retrospect on stage models of e-Government: A qualitative meta-synthesis. Government Information Quarterly, 27(3), 220-230.

Linders, D. (2012). From e-government to we-government: Defining a typology for citizen coproduction in the age of social media. Government Information Quarterly, 29(4), 446-454.

Lofstedt, U. (2012). E-government-assesment of current research and some proposals for future directions. International journal of public information systems, 1(1).

Malik, M. B., Ghazi, M. A., \& Ali, R. (2012). Privacy preserving data mining techniques: current scenario and future prospects. Paper presented at the 2012 3rd International Conference on Computer and Communication Technology (ICCCT 2012).

Saha, P. (2012). Connected government as the new normal: A strategic thinking approach to whole-ofgovernment enterprise architecture adoption. Enterprise Architecture for Connected EGovernment: Practices and Innovations (pp. 1-55): IGI Global.

Siau, K., \& Long, Y. (2005). Synthesizing e-government stage models- a meta-synthesis based on metaethnography approach. Industrial Management \& Data Systems, 105(4), 443-458.

Winter, R., \& Fischer, R. (2006). Essential layers, artifacts, and dependencies of enterprise architecture. Paper presented at the Enterprise Distributed Object Computing Conference Workshops, 2006. EDOCW'06. 10th IEEE International.

Wyld, D. C. (2010). The cloudy future of government IT: Cloud computing and the public sector around the world. International J ournal of Web \& Semantic Technology, 1(1), 1-20. 